\title{
Constrained Dynamic Optimality and Binomial Terminal Wealth
}

DOI:

10.1137/16M1085097

\section{Document Version}

Accepted author manuscript

Link to publication record in Manchester Research Explorer

\section{Citation for published version (APA):}

Pedersen, J.L., \& Peskir, G. (2018). Constrained Dynamic Optimality and Binomial Terminal Wealth. SIAM Journal on Control and Optimization, 56(2), 1342-1357. https://doi.org/10.1137/16M1085097

\section{Published in:}

SIAM Journal on Control and Optimization

\section{Citing this paper}

Please note that where the full-text provided on Manchester Research Explorer is the Author Accepted Manuscript or Proof version this may differ from the final Published version. If citing, it is advised that you check and use the publisher's definitive version.

\section{General rights}

Copyright and moral rights for the publications made accessible in the Research Explorer are retained by the authors and/or other copyright owners and it is a condition of accessing publications that users recognise and abide by the legal requirements associated with these rights.

\section{Takedown policy}

If you believe that this document breaches copyright please refer to the University of Manchester's Takedown Procedures [http://man.ac.uk/04Y6Bo] or contact uml.scholarlycommunications@manchester.ac.uk providing relevant details, so we can investigate your claim.

\section{OPEN ACCESS}




\title{
Constrained Dynamic Optimality and Binomial Terminal Wealth
}

\author{
J. L. Pedersen \& G. Peskir
}

\begin{abstract}
We assume that the wealth process $X^{u}$ is self-financing and generated from the initial wealth by holding a fraction $u$ of $X^{u}$ in a risky stock (whose price follows a geometric Brownian motion) and the remaining fraction $1-u$ of $X^{u}$ in a riskless bond (whose price compounds exponentially with interest rate $r \in \mathbb{R}$ ). Letting $\mathrm{P}_{t, x}$ denote a probability measure under which $X^{u}$ takes value $x$ at time $t$, we study the dynamic version of the nonlinear optimal control problem

$$
\inf _{u} \operatorname{Var}_{t, X_{t}^{u}}\left(X_{T}^{u}\right)
$$

where the infimum is taken over admissible controls $u$ subject to $X_{t}^{u} \geq e^{-r(T-t)} g$ and $\mathrm{E}_{t, X_{t}^{u}}\left(X_{T}^{u}\right) \geq \beta$ for $t \in[0, T]$. The two constants $g$ and $\beta$ are assumed to be given exogenously and fixed. By conditioning on the expected terminal wealth value, we show that the nonlinear problem can be reduced to a family of linear problems. Solving the latter using a martingale method combined with Lagrange multipliers, we derive the dynamically optimal control $u_{*}^{d}$ in closed form and prove that the dynamically optimal terminal wealth $X_{T}^{d}$ can only take two values $g$ and $\beta$. This binomial nature of the dynamically optimal strategy stands in sharp contrast with other known portfolio selection strategies encountered in the literature. A direct comparison shows that the dynamically optimal (time-consistent) strategy outperforms the statically optimal (time-inconsistent) strategy in the problem.
\end{abstract}

\section{Introduction}

Imagine an investor who has an initial wealth which he wishes to exchange between a risky stock and a riskless bond, in a self-financing manner, dynamically in time, so as to minimise his risk in obtaining a desired return at the given terminal time. In line with the meanvariance analysis of Markowitz [14], where the optimal portfolio selection problem of this kind was solved in a single period model (see e.g. Merton [15] and the references therein), we will identify the return with the expectation of the terminal wealth, and the risk with the variance of the terminal wealth. The quadratic nonlinearity of the variance then moves the resulting optimal control problem outside the scope of the standard optimal control theory, which yields optimal controls dependent on the initial time/wealth, and hence time inconsistent (see [16] for further details and a historical review dating back to Strotz [20]).

A new methodology for solving nonlinear control problems of this kind has been recently developed in [16]. Its central idea rests on the concept of dynamic optimality which consists of

Mathematics Subject Classification 2010. Primary 60H30, 60J65. Secondary 91G80, 93E20.

Key words and phrases: Constrained nonlinear optimal control, dynamic optimality, static optimality, mean-variance analysis, martingale, Lagrange multiplier, geometric Brownian motion, Markov process. 
continuous rebalancing of optimal controls upon overruling all the past controls. This makes dynamically optimal controls time consistent. It stands in contrast with the statically optimal (pre-committed) controls addressed above which are time inconsistent. For more details including comparisons with the subgame-perfect Nash equilibrium controls (dating back to the concept of 'consistent planning' in Strotz [20]) we refer to [16] and the references therein.

Assuming that the stock price follows a geometric Brownian motion and the bond price compounds exponentially, in [16] we derived the dynamically optimal controls for the investor aiming to minimise the variance of his terminal wealth $X_{T}^{u}$ over all admissible controls $u$ (representing the fraction of the wealth held in the stock) such that the expectation of $X_{T}^{u}$ is bounded below by a given constant $\beta$. We showed in [16, Corollary 7] that the dynamically optimal wealth process $X^{d}$ solves a meander type equation which makes $X^{d}$ hit $\beta$ exactly at the terminal time $T$ (not before). This was established without any pathwise constraints on the wealth process which could take low/negative values of unlimited size, leading to a potential bankruptcy, and thus making the trading strategy less attractive to the investor.

Motivated by this deficiency, in this paper we consider the analogous variance minimising problem obtained upon imposing the guarantee (stop-loss rule) that the wealth process always stays above a given constant $g$, regardless of whether the investment is favourable or not. In addition to finance, where $g=0$ corresponds to bankruptcy, such constraints are also widely used in economics (see e.g. [3] and the references therein). By conditioning on the expected terminal wealth value we show that the nonlinear problem can be reduced to a family of linear problems. Solving the latter using a martingale method (cf. Pliska [17]) combined with Lagrange multipliers (cf. White [21]) we derive the dynamically optimal control $u_{*}^{d}$ in closed form and establish that the dynamically optimal wealth process $X^{d}$ can be characterised as the unique (strong) solution to a stochastic differential equation with time-dependent coefficients. By analysing this stochastic differential equation (extending Feller's test to time-inhomogeneous diffusions that is of independent interest) we prove that the dynamically optimal terminal wealth $X_{T}^{d}$ can only take two values $g$ and $\beta$. This binomial nature of the dynamically optimal strategy stands in sharp contrast with other known portfolio selection strategies encountered in the literature. A direct comparison shows that the dynamically optimal (time-consistent) strategy outperforms the statically optimal (time-inconsistent) strategy in the minimising variance problem.

The portfolio selection problem of minimising the variance of the terminal wealth given its expected value has been firstly studied by Richardson [18] without imposing pathwise constraints on low/negative wealth. Lagrange reformulations of this problem were studied in [12] (discrete time) and [22] (continuous time) without imposing pathwise constraints on low/negative wealth either (see also [13]). Korn and Trautmann [11] studied Richardson's problem under a pathwise constraint on low/negative wealth using duality techniques (see also [7] and [10]). Bielecki at al [5] extended these results to a more general setting using backward stochastic differential equations (see also [4] for applications in insurance mathematics).

The portfolio selection strategies in all these papers are, as pointed out by Basak and Chabakauri [1], pre-committed (statically optimal) and hence time inconsistent (see also [2]). The dynamically optimal strategies derived in the present paper are, to our knowledge, the first known optimal strategies that are time consistent in the variance minimising problem with pathwise constraints on low/negative wealth. It appears to be a challenging problem to derive such strategies in the context of the subgame-perfect Nash equilibrium and any comparison with other time-consistent strategies is currently unavailable. 


\section{Formulation of the problem}

Assume that the riskless bond price $B$ solves

$$
d B_{t}=r B_{t} d t
$$

with $B_{0}=b$ for some $b>0$ where $r \in \mathbb{R}$ is the interest rate. Let the risky stock price $S$ follow a geometric Brownian motion solving

$$
d S_{t}=\mu S_{t} d t+\sigma S_{t} d W_{t}
$$

with $S_{0}=s$ for some $s>0$, where $\mu \in \mathbb{R}$ is the drift, $\sigma>0$ is the volatility, and $W$ is a standard Brownian motion defined on a probability space $(\Omega, \mathcal{F}, \mathrm{P})$. Note that a unique solution to (2.1) is given by $B_{t}=b e^{r t}$ and recall that a unique strong solution to (2.2) is given by $S_{t}=s \exp \left(\sigma W_{t}+\left(\mu-\left(\sigma^{2} / 2\right)\right) t\right)$ for $t \geq 0$.

Consider the investor who has an initial wealth $x_{0} \in \mathbb{R}$ which he wishes to exchange between $B$ and $S$ in a self-financing manner (with no exogenous infusion or withdrawal of wealth) dynamically in time up to the given horizon $T>0$. It is then well known (see e.g. [6, Chapter 6]) that the investor's wealth $X^{u}$ solves

$$
d X_{t}^{u}=\left(r\left(1-u_{t}\right)+\mu u_{t}\right) X_{t}^{u} d t+\sigma u_{t} X_{t}^{u} d W_{t}
$$

with $X_{t_{0}}^{u}=x_{0}$ where $u_{t}$ denotes the fraction of the investor's wealth held in the stock at time $t \in\left[t_{0}, T\right]$ for $t_{0} \in[0, T)$ given and fixed. Note that (i) $u_{t}<0$ corresponds to short selling of the stock, (ii) $u_{t}>1$ corresponds to borrowing from the bond, and (iii) $u_{t} \in[0,1]$ corresponds to a long position in both the stock and the bond.

To simplify the exposition, we will assume that the control $u$ in (2.3) is given by $u_{t}=$ $u\left(t, X_{t}^{u}\right)$ where $(t, x) \mapsto u(t, x) \cdot x$ is a continuous function from $[0, T] \times \mathbb{R}$ into $\mathbb{R}$ for which the stochastic differential equation (2.3) understood in Itô's sense has a unique strong solution $X^{u}$ (meaning that the solution $X^{u}$ to (2.3) is adapted to the natural filtration of $W$, and if $\tilde{X}^{u}$ is another solution to (2.3) of this kind, then $X^{u}$ and $\tilde{X}^{u}$ are equal almost surely). We will call controls of this kind admissible in the sequel. For more general controls, yielding the same solution to the problem to be presented below, we refer to [16, Section 2] and the references therein. Recalling that the natural filtration of $S$ coincides with the natural filtration of $W$, we see that admissible controls have a natural financial interpretation, as they are obtained as deterministic (measurable) functionals of the observed stock price. We will denote the natural filtration by $\left(\mathcal{F}_{t}\right)_{0 \leq t \leq T}$ in the sequel (omitting superscripts $S$ or $W$ for simplicity).

For a given admissible control $u$ we let $\mathrm{P}_{t, x}$ denote the probability measure under which the solution $X^{u}$ to (2.3) takes value $x$ at time $t$ for $(t, x) \in[0, T] \times \mathbb{R}$. Note that $X^{u}$ is a (strong) Markov process with respect to $\mathrm{P}_{t, x}$ for $(t, x) \in[0, T] \times \mathbb{R}$. To formulate the investor's problem, we consider admissible controls $u$ obeying the pathwise constraint

$$
X_{s}^{u} \geq e^{-r(T-s)} g \quad \mathrm{P}_{t, x^{-}} \text {a.s. for all } s \in[t, T]
$$

where $g$ is a given constant playing the financial role of a guarantee (not to be strictly crossed from above). This is a primary constraint on the investor's wealth process. Note that if $x=e^{-r(T-t)} g$ then the only admissible control $u$ which can meet the primary constraint (2.4) 
equals 0 (note also that if $x<e^{-r(T-t)} g$ then no admissible control can meet the constraint). This corresponds to investing all the wealth in the bond over the remaining time interval $[t, T]$. If $x \in\left(e^{-r(T-t)} g, e^{-r(T-t)} \beta\right.$ ) for a given constant $\beta$ (strictly larger than $g$ ), we also impose a secondary constraint on the investor's wealth process which is expressed as

$$
\mathrm{E}_{t, x}\left(X_{T}^{u}\right) \geq \beta
$$

where $\beta$ plays the financial role of a target (to be met at the terminal time $T$ ). Note that if $x \geq e^{-r(T-t)} \beta$ then the admissible control $u$ which meets the primary constraint (2.4) and the secondary constraint (2.5) equals 0 . This again corresponds to investing all the wealth in the bond with zero variance (risk) at the terminal time $T$. For these reasons we will see from the problem formulation below that the only interesting/nontrivial case is when $x \in\left(e^{-r(T-t)} g, e^{-r(T-t)} \beta\right)$ for $(t, x) \in[0, T] \times \mathbb{R}$. Given that we are interested in admissible controls yielding minimal terminal risk (identified with variance), there is no loss of generality in assuming that the terminal wealth of each admissible control is square-integrable. A sufficient condition for the latter expectation to be finite is that $\mathrm{E}_{t, x}\left[\int_{t}^{T}\left(1+u_{s}^{2}\right)\left(X_{s}^{u}\right)^{2} d s\right]<\infty$ for $(t, x) \in[0, T] \times \mathbb{R}$. The imposed requirements may now be summarised as follows.

Definition 1 (Pathwise constrained strategies). The family of admissible strategies $u$ satisfying the primary constraint (2.4) with the secondary constraint (2.5) (whenever feasible) and the integrability condition $\mathrm{E}_{t, x}\left[\int_{t}^{T}\left(1+u_{s}^{2}\right)\left(X_{s}^{u}\right)^{2} d s\right]<\infty$ for $(t, x) \in[0, T] \times \mathbb{R}$ will be denoted by $C_{g, \beta}$ where $g<\beta$ are given and fixed constants.

Having defined pathwise constrained strategies, consider the optimal control problem

$$
V(t, x)=\inf _{u \in C_{g, \beta}} \operatorname{Var}_{t, x}\left(X_{T}^{u}\right)
$$

for $(t, x) \in[0, T] \times \mathbb{R}$ with $g<\beta$ given and fixed. Recall that we can focus on the case when $x \in\left(e^{-r(T-t)} g, e^{-r(T-t)} \beta\right)$ for $(t, x) \in[0, T] \times \mathbb{R}$ as other cases are evident. The problem (2.6) seeks to minimise the investor's risk (identified with the variance of $X_{T}^{u}$ ), upon applying a control $u$ which yields a desired investor's return (identified with the expectation of $X_{T}^{u}$ ), and keeps the investor's wealth above a desired/guaranteed threshold at all times by the terminal time $T$. This identification is done in line with the mean-variance analysis of Markowitz [14]. A (time-consistent) solution to the problem (2.6) is known in the pathwise unconstrained case corresponding to no guarantee (see [16, Corollary 7]). The main purpose of the present paper is to derive a (time-consistent) solution in the pathwise constrained case.

Due to the quadratic nonlinearity of the second term in the expression $\operatorname{Var}_{t, x}\left(X_{T}^{u}\right)=$ $\mathrm{E}_{t, x}\left[\left(X_{T}^{u}\right)^{2}\right]-\left[\mathrm{E}_{t, x}\left(X_{T}^{u}\right)\right]^{2}$ it is known that the problem (2.6) falls outside the scope of the standard/linear optimal control theory for Markov processes. In addition to the static (precommitted) formulation of the nonlinear problem (2.6), where the minimisation takes place relative to the initial point $(t, x)$ which is given and fixed (cf. [16, Definition 1]), one is also naturally led to consider a dynamic formulation of the nonlinear problem (2.6), in which each new position of the controlled process $\left(\left(t, X_{t}^{u}\right)\right)_{t \in[0, T]}$ yields a new optimal control problem, to be solved upon overruling all the past problems (cf. [16, Definition 2]).

For the sake of completeness we now recall the definition of dynamic optimality for pathwise constrained strategies in $C_{g, \beta}$. Recall that all controls throughout refer to admissible controls as defined/discussed above. 
Definition 2 (Dynamic optimality). A control $u_{*}$ is dynamically optimal in (2.6), if for every given and fixed $(t, x) \in[0, T) \times \mathbb{R}$ and every control $v$ from $C_{g, \beta}$ such that $v(t, x) \neq u_{*}(t, x)$, there exists a control $w$ in $C_{g, \beta}$ satisfying $w(t, x)=u_{*}(t, x)$ such that

$$
\operatorname{Var}_{t, x}\left(X_{T}^{w}\right)<\operatorname{Var}_{t, x}\left(X_{T}^{v}\right)
$$

Dynamic optimality above is understood in the 'strong' sense. Replacing the strict inequality in (2.7) by an inequality would yield dynamic optimality in the 'weak' sense.

Note that the dynamic optimality corresponds to solving infinitely many optimal control problems dynamically in time, where each new position of the controlled process $\left(\left(t, X_{t}^{u}\right)\right)_{t \in[0, T]}$ yields a new optimal control problem, to be solved upon overruling all the past problems. In effect, this corresponds to continuous rebalancing of optimal controls, upon overruling all the past controls, which makes the dynamically optimal controls time consistent (cf. [16]).

\section{Solution to the problem}

To formulate the main results below we introduce the following notation:

$$
\begin{aligned}
& L_{t}=e^{-\delta W_{t}-\frac{\delta^{2}}{2} t} \quad \& \quad H_{t}=e^{-r t} L_{t} \\
& F_{0}(T-t, \lambda):=E\left[\left(\lambda-H_{T-t}\right)^{+}\right]=\lambda \Phi\left(\frac{\log \lambda+\left(r+\frac{\delta^{2}}{2}\right)(T-t)}{|\delta| \sqrt{T-t}}\right) \\
& -e^{-r(T-t)} \Phi\left(\frac{\log \lambda+\left(r-\frac{\delta^{2}}{2}\right)(T-t)}{|\delta| \sqrt{T-t}}\right) \\
& F_{1}(T-t, \lambda):=E\left[H_{T-t}\left(\lambda-H_{T-t}\right)^{+}\right]=\lambda e^{-r(T-t)} \Phi\left(\frac{\log \lambda+\left(r-\frac{\delta^{2}}{2}\right)(T-t)}{|\delta| \sqrt{T-t}}\right) \\
& -e^{\left(\delta^{2}-2 r\right)(T-t)} \Phi\left(\frac{\log \lambda+\left(r-\frac{3 \delta^{2}}{2}\right)(T-t)}{|\delta| \sqrt{T-t}}\right) \\
& F_{2}(T-t, \lambda):=E\left[H_{T-t}^{2} I\left(\lambda>H_{T-t}\right)\right]=e^{\left(\delta^{2}-2 r\right)(T-t)} \Phi\left(\frac{\log \lambda+\left(r-\frac{3 \delta^{2}}{2}\right)(T-t)}{|\delta| \sqrt{T-t}}\right) \\
& F(T-t, \lambda):=\frac{F_{1}(T-t, \lambda)}{F_{0}(T-t, \lambda)}
\end{aligned}
$$

for $t \in[0, T]$ and $\lambda>0$ where the expressions on the right-hand side of (3.2)-(3.4) are derived using standard arguments (recall that $\Phi(x)=(1 / \sqrt{2 \pi}) \int_{-\infty}^{x} e^{-y^{2} / 2} d y$ denotes the standard normal distribution function for $x \in \mathbb{R})$.

Theorem 3. Consider the optimal control problem (2.6) where $X^{u}$ solves (2.3) with $X_{t_{0}}^{u}=x_{0}$ under $\mathrm{P}_{t_{0}, x_{0}}$ for $\left(t_{0}, x_{0}\right) \in[0, T] \times \mathbb{R}$ given and fixed. Recall that $B$ solves (2.1), $S$ solves (2.2), and we set $\delta=(\mu-r) / \sigma$ for $\mu \in \mathbb{R}, r \in \mathbb{R}$ and $\sigma>0$. We assume throughout that $\delta \neq 0$ to exclude the trivial case (the case $\delta=0$ follows by passage to the limit when the non-zero $\delta$ tends to 0 ). 
(A) The dynamically optimal control $u_{*}^{d}$ in (2.6) is given by

$$
u_{*}^{d}(t, x)=\frac{\delta}{\sigma} \frac{1}{x}\left(x-e^{-r(T-t)} g\right) a(T-t, x)
$$

where the function a is defined by

$$
a(T-t, x)=\frac{F_{2}(T-t, \lambda(T-t, x))}{F_{1}(T-t, \lambda(T-t, x))}
$$

and $\lambda=\lambda(T-t, x)$ is the unique strictly positive solution to the equation

$$
F(T-t, \lambda)=\frac{x-e^{-r(T-t)} g}{\beta-g}
$$

for $(t, x) \in\left[t_{0}, T\right] \times \mathbb{R}$ such that $e^{-r(T-t)} g<x<e^{-r(T-t)} \beta$. If $x=e^{-r(T-t)} g$ or $x \geq e^{-r(T-t)} \beta$ then $u_{*}^{d}(t, x)=0$ (as discussed prior to Definition 1 above).

(B) The dynamically optimal controlled process $X^{d}$ in (2.6) can be characterised as the unique strong solution to the stochastic differential equation

$$
d X_{t}^{d}=\left[r X_{t}^{d}+\delta^{2}\left(X_{t}^{d}-e^{-r(T-t)} g\right) a\left(T-t, X_{t}^{d}\right)\right] d t+\delta\left(X_{t}^{d}-e^{-r(T-t)} g\right) a\left(T-t, X_{t}^{d}\right) d W_{t}
$$

satisfying $X_{t_{0}}^{d}=x_{0} \in\left(e^{-r\left(T-t_{0}\right)} g, e^{-r\left(T-t_{0}\right)} \beta\right)$, until the first exit time of $X_{t}^{d}$ from the interval $\left(e^{-r(T-t)} g, e^{-r(T-t)} \beta\right)$, when $t$ runs from $t_{0}$ to $T$, followed by the constant motion (at its left-hand boundary) corresponding to $u_{*}^{d}=0$ afterwards. Setting

$$
\begin{aligned}
& \tau_{\beta}=\inf \left\{t \in\left[t_{0}, T\right] \mid X_{t}^{d}=e^{-r(T-t)} \beta\right\} \\
& \tau_{g}=\inf \left\{t \in\left[t_{0}, T\right] \mid X_{t}^{d}=e^{-r(T-t)} g\right\}
\end{aligned}
$$

the following (binomial) relations are satisfied

$$
\begin{gathered}
\mathrm{P}_{t_{0}, x_{0}}\left(\tau_{\beta}<T\right)=0 \& \mathrm{P}_{t_{0}, x_{0}}\left(\tau_{\beta}=T\right)>0 \\
\mathrm{P}_{t_{0}, x_{0}}\left(\tau_{g}<T\right)>0
\end{gathered}
$$

This means that the dynamically optimally controlled process $X^{d}$ (i) hits the target $\beta$ exactly at the terminal time $T$ (never before $T$ ) with a strictly positive probability, and (ii) hits the guarantee $g$ strictly before the terminal time $T$ with a strictly positive probability (see Figure 1 below).

Proof. We assume throughout that the process $X^{u}$ solves the stochastic differential equation (2.3), with $X_{t_{0}}^{u}=x_{0}$ under $\mathrm{P}_{t_{0}, x_{0}}$ for $\left(t_{0}, x_{0}\right) \in[0, T] \times \mathbb{R}$ given and fixed, where $u$ is any admissible control as defined/discussed above.

(A): Recall that the optimal control problem (2.6) seeks to minimise

$$
\operatorname{Var}_{t_{0}, x_{0}}\left(X_{T}^{u}\right)=\mathrm{E}_{t_{0}, x_{0}}\left[\left(X_{T}^{u}\right)^{2}\right]-\left[\mathrm{E}_{t_{0}, x_{0}}\left(X_{T}^{u}\right)\right]^{2}
$$

over all admissible controls $u$ subject to the primary (pathwise) constraint

$$
X_{t}^{u} \geq e^{-r(T-t)} g \quad \mathrm{P}_{t_{0}, x_{0}} \text {-a.s. for all } t \in\left[t_{0}, T\right]
$$



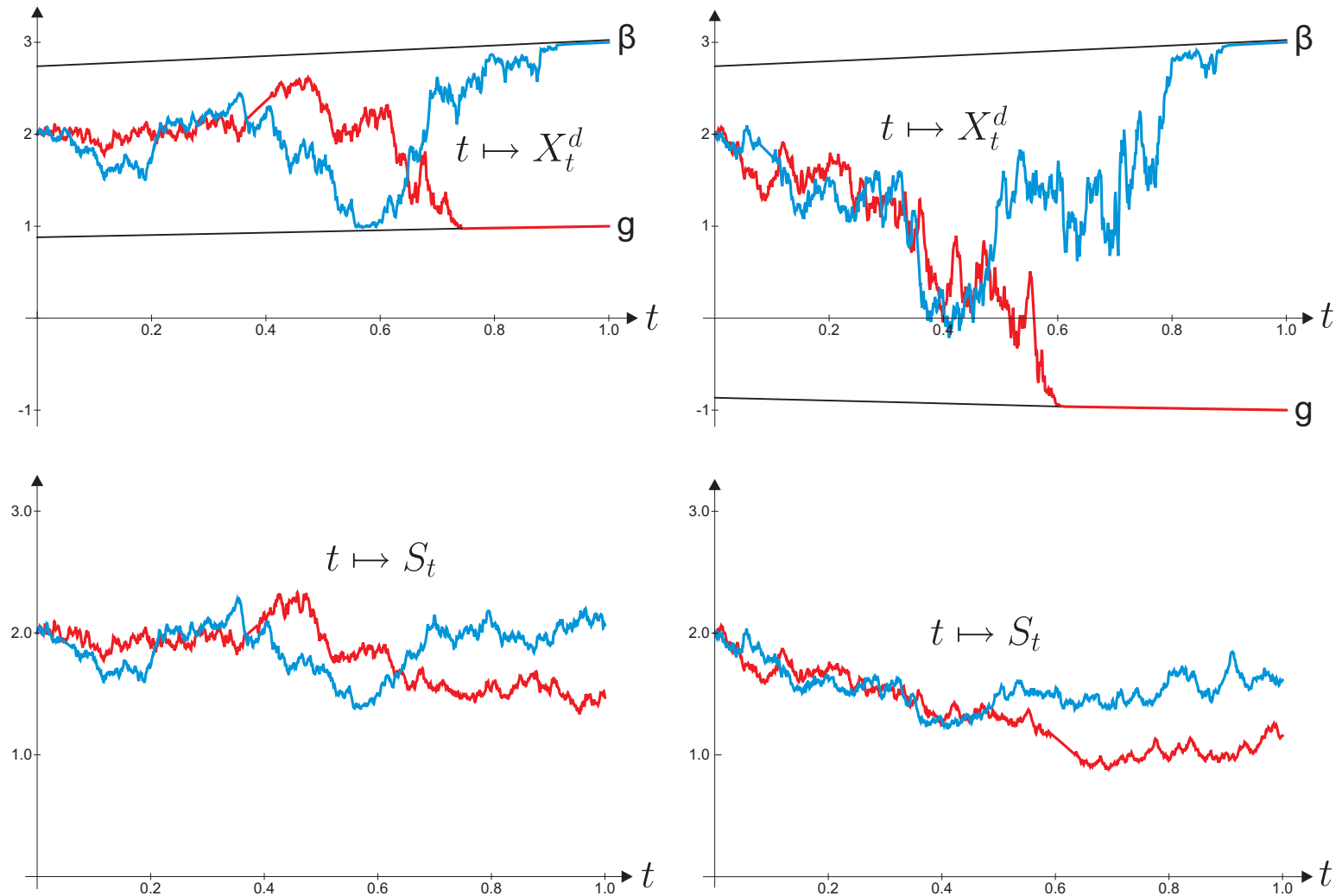

Figure 1. The dynamically optimal wealth $t \mapsto X_{t}^{d}$ in the constrained problem (2.6) of Theorem 3 obtained from the stock price $t \mapsto S_{t}$ when $t_{0}=0, x_{0}=2, S_{0}=2, \beta=3$, $r=0.1, \mu=0, \sigma=0.4, T=1, g=1$ (left-hand graphs) and $g=-1$ (right-hand graphs). Note that the expected value of $S_{T}$ equals 2 which is strictly smaller than $\beta$.

and the secondary (mean value) constraint

$$
\mathrm{E}_{t_{0}, x_{0}}\left(X_{T}^{u}\right) \geq \beta
$$

as specified in Definition 1 above.

1. Using (3.1) and passing to the equivalent martingale measure defined by

$$
d \tilde{\mathrm{P}}_{t_{0}, x_{0}}:=\left(L_{T} / L_{t_{0}}\right) d \mathrm{P}_{t_{0}, x_{0}}
$$

under which $\tilde{W}_{t}:=W_{t}-W_{t_{0}}+\delta\left(t-t_{0}\right)$ is a standard Brownian motion for $t \in\left[t_{0}, T\right]$, and recalling that the discounted wealth process defined by

$$
\tilde{X}_{t}^{u}:=e^{-r\left(t-t_{0}\right)} X_{t}^{u}
$$

solves the stochastic differential equation

$$
d \tilde{X}_{t}^{u}=\sigma u_{t} \tilde{X}_{t}^{u} d \tilde{W}_{t}
$$


and defines a martingale for $t \in\left[t_{0}, T\right]$, one sees that the pathwise constraint (3.15) is equivalent to the (seemingly weaker) terminal constraint

$$
X_{T}^{u} \geq g \quad \mathrm{P}_{t_{0}, x_{0}} \text {-a.s. }
$$

Indeed, while (3.15) evidently implies (3.20), to see that the converse is also true, it is sufficient to apply the martingale property of $\tilde{X}^{u}$ (cf. [16, Remark 9]) which by means of (3.20) yields

$$
e^{-r\left(t-t_{0}\right)} X_{t}^{u}=\tilde{X}_{t}^{u}=\tilde{\mathrm{E}}_{t_{0}, x_{0}}\left(\tilde{X}_{T}^{u} \mid \mathcal{F}_{t}\right) \geq e^{-r\left(T-t_{0}\right)} g
$$

and implies (3.15) as claimed. By the martingale property of $\tilde{X}^{u}$ we also have

$$
\tilde{\mathrm{E}}_{t_{0}, x_{0}}\left(\tilde{X}_{T}^{u}\right)=x_{0}
$$

which can be rewritten using (3.17) and (3.18) as follows

$$
\mathrm{E}_{t_{0}, x_{0}}\left[\left(H_{T} / H_{t_{0}}\right) X_{T}^{u}\right]=x_{0}
$$

showing that admissible controls $u$ stand in one-to-one correspondence with $\mathcal{F}_{T}$-measurable square-integrable random variables $X_{T}^{u}$ satisfying (3.23). In the latter conclusion we use the Itô-Clark theorem (see e.g. [9, pp 185-189]) applied to the stochastic integral with respect to $\tilde{W}$ over $\left[t_{0}, T\right]$ under $\tilde{\mathrm{P}}_{t_{0}, x_{0}}$. Details of this will be utilised further in the proof below.

2. The previous considerations show that the optimal control problem (3.14) is equivalent to the pointwise constrained optimisation problem

$$
\inf _{X \geq g} \mathrm{E}\left(X^{2}\right)
$$

subject to the mean value constraints

$$
\mathrm{E}(X)=\beta \quad \& \quad \mathrm{E}(H X)=x
$$

where we impose equality in (3.16) and simplify the notation by setting $X:=X_{T}^{u}, \mathrm{P}:=\mathrm{P}_{t_{0}, x_{0}}$, $H:=H_{T} / H_{t_{0}}$ and $x=x_{0}$ (we will return to the original notation below).

3. To solve the problem (3.24)-(3.25) we optimise the Lagrangian

$$
\inf _{X \geq g} \mathrm{E}\left(X^{2}-\lambda_{1} X-\lambda_{2} H X\right)
$$

for $\lambda_{1}$ and $\lambda_{2}$ in $\mathbb{R}$ to be determined. Pointwise minimisation of the expression under the expectation sign in (3.26) then yields the following (unique) solution

$$
X_{*}=\frac{1}{2}\left(\lambda_{1}+\lambda_{2} H\right) \vee g=\left(\frac{\lambda_{1}}{2}+\frac{\lambda_{2}}{2} H-g\right)^{+}+g=\kappa(\lambda-H)^{+}+g
$$

upon setting $\kappa=-\lambda_{2} / 2$ and $\lambda=\left(\lambda_{1} / 2-g\right) /\left(-\lambda_{2} / 2\right)$, where we impose $\kappa>0$ i.e. $\lambda_{2}<0$ (see Remark 4 below for fuller details). Using (3.25) we get

$$
\begin{aligned}
& \beta=E\left(X_{*}\right)=\kappa \mathrm{E}\left[(\lambda-H)^{+}\right]+g \\
& x=E\left(H X_{*}\right)=\kappa \mathrm{E}\left[H(\lambda-H)^{+}\right]+g E(H) .
\end{aligned}
$$


From (3.28) and (3.29) we find that

$$
\kappa=\frac{\beta-g}{\mathrm{E}\left[(\lambda-H)^{+}\right]}=\frac{x-g E(H)}{\mathrm{E}\left[H(\lambda-H)^{+}\right]} .
$$

The second identity in (3.30) can be rewritten as follows

$$
\frac{\mathrm{E}\left[H(\lambda-H)^{+}\right]}{\mathrm{E}\left[(\lambda-H)^{+}\right]}=\frac{x-g E(H)}{\beta-g} .
$$

4. We now show that there exists a unique $\lambda>0$ solving (3.31) when $x \in(g \mathrm{E}(H), \beta \mathrm{E}(H))$ and $\mathrm{P}(0<|H| \leq \varepsilon)>0$ for each $\varepsilon>0$ (assuming also that $E(H)$ exists and is finite).

For this, define the function

$$
F(\lambda):=\frac{\mathrm{E}\left[H(\lambda-H)^{+}\right]}{\mathrm{E}\left[(\lambda-H)^{+}\right]}
$$

for $\lambda>0$. We then claim that

$$
\begin{aligned}
& \lambda \mapsto F(\lambda) \text { is strictly increasing on }(0, \infty) \\
& F(0+)=0 \quad \& \quad F(+\infty)=E(H) .
\end{aligned}
$$

To verify (3.33) it is enough to show that $F^{\prime}(\lambda)>0$ for all $\lambda>0$ such that $\mathrm{P}(H=\lambda)=0$ (recall that there could be at most countably many $\lambda>0$ for which this fails). Taking any such $\lambda>0$ and differentiating under the expectation sign in (3.32) (which is easily justified using standard means) we see that $F^{\prime}(\lambda)>0$ and if and only if

$$
\mathrm{E}[H I(\lambda>H)] \mathrm{E}[(\lambda-H) I(\lambda>H)]>\mathrm{E}[H(\lambda-H) I(\lambda>H)] \mathrm{P}(\lambda>H)
$$

which in turn is equivalent to

$$
(\mathrm{E}[H I(\lambda>H)])^{2}<\mathrm{E}\left[H^{2} I(\lambda>H)\right] \mathrm{P}(\lambda>H) .
$$

Writing the integrand on the left-hand side as $H I(\lambda>H)=H I(\lambda>H) \cdot I(\lambda>H)$ we recognise (3.36) as the Hölder inequality. It is well known (see [8, Exc. 3.35]) that equality holds in the Hölder inequality if and only if $|H| I(\lambda>H)=c I(\lambda>H)$ for some $c \geq 0$. Hence if the strict inequality in (3.36) would not hold, this would violate the hypotheses that $\mathrm{P}(0<|H| \leq \varepsilon)>0$ for all $\varepsilon>0$. It follows therefore that (3.33) holds as claimed.

The first equality in (3.34) follows from the fact that $F(\lambda) \leq \lambda$ for all $\lambda>0$ and the second equality in (3.34) follows by noting that $F(\lambda)=\mathrm{E}\left[H(1-H / \lambda)^{+}\right] / E\left[(1-H / \lambda)^{+}\right]$and using the dominated converge theorem upon letting $\lambda \rightarrow \infty$. Noting that $F(\lambda)$ equals the left-hand side in (3.31) and that $\lambda \mapsto F(\lambda)$ is continuous on $(0, \infty)$, we see by $(3.33)+(3.34)$ that there exists a unique $\lambda>0$ solving (3.31) as claimed.

5. The existence of a unique $\lambda>0$ solving (3.31) combined with (3.30) shows that $X_{*}$ defined in (3.27) is the solution to the constrained problem (3.24)+(3.25). Recalling that $X:=X_{T}^{u}, \mathrm{P}:=\mathrm{P}_{t_{0}, x_{0}}, H:=H_{T} / H_{t_{0}}$ and $x=x_{0}$, we see that the equation (3.31) is the 
same as the equation (3.8) with $x=x_{0}$ in the statement of the theorem. We will return to this point later in the proof. Instead we now turn to finding the statically optimal control $u_{*}^{s}$ and its wealth process $X^{s}$ which yields $X_{*}$ at the terminal time $T$.

6. To find the statically optimal control $u_{*}^{s}$ and its wealth process $X^{s}$ satisfying $X_{T}^{s}=X_{*}$, we can exploit the martingale property of $\tilde{X}^{s}$ defined as in (3.18) with $u=u_{*}^{s}$, which in view of (3.27) yields the following identities

$$
X_{t}^{s}=e^{-r(T-t)} \tilde{E}_{t_{0}, x_{0}}\left(X_{*} \mid \mathcal{F}_{t}\right)=e^{-r(T-t)}\left[\kappa \tilde{E}_{t_{0}, x_{0}}\left(\left(\lambda-H_{T} / H_{t_{0}}\right)^{+} \mid \mathcal{F}_{t}\right)+g\right]
$$

for $t \in\left[t_{0}, T\right]$, where $\lambda=\lambda\left(T-t_{0}, x_{0}\right)$ is the unique strictly positive solution to (3.31), and $\kappa=\kappa\left(T-t_{0}, x_{0}\right)$ is given by (3.30) with $\mathrm{P}=\mathrm{P}_{t_{0}, x_{0}}, H=H_{T} / H_{t_{0}}$ and $x=x_{0}$. Recalling (3.1) we see that $\tilde{H}_{t}:=H_{t} / H_{t_{0}}$ solves

$$
d \tilde{H}_{t}=\left(\delta^{2}-r\right) \tilde{H}_{t} d t-\delta \tilde{H}_{t} \tilde{W}_{t}
$$

and defines a geometric Brownian motion under $\tilde{\mathrm{P}}_{t_{0}, x_{0}}$ for $t \in\left[t_{0}, T\right]$. Writing $H_{T} / H_{t_{0}}$ in (3.37) as $\left(H_{t} / H_{t_{0}}\right)\left(H_{T} / H_{t}\right)$, and using independent increments of $\tilde{W}$, we see that (3.37) reads

$$
X_{t}^{s}=e^{-r(T-t)}\left[\kappa P\left(T-t, \tilde{H}_{t}\right)+g\right]
$$

where the function $P$ is defined by

$$
\begin{aligned}
P(T-t, h) & =\tilde{E}_{t_{0}, x_{0}}\left[\left(\lambda-h\left(H_{T} / H_{t}\right)\right)^{+}\right]=\mathrm{E}_{t_{0}, x_{0}}\left[\frac{L_{T}}{L_{t}} \frac{L_{t}}{L_{t_{0}}}\left(\lambda-h\left(H_{T} / H_{t}\right)\right)^{+}\right] \\
& =e^{r(T-t)} \mathrm{E}\left[H_{T-t}\left(\lambda-h H_{T-t}\right)^{+}\right]
\end{aligned}
$$

for $t \in\left[t_{0}, T\right]$, and in the final equality we use that $L_{t} / L_{t_{0}}$ is $\mathcal{F}_{t}$-measurable (with mean equal one) and both $L_{T} / L_{t}$ and $H_{T} / H_{t}$ are independent from $\mathcal{F}_{t}$. Note that the second identity in (3.3) can be used to express $P(T-t, h)$ in closed form using the standard normal distribution function $\Phi$, however, we will omit this explicit expression for simplicity.

From (3.39) we see that

$$
Z_{t}^{s}:=e^{r(T-t)} X_{t}^{s}=\kappa P\left(T-t, \tilde{H}_{t}\right)+g
$$

for $t \in\left[t_{0}, T\right]$. Aplying Itô's formula to the right-hand side of (3.41) and using that $Z^{s}$ is a martingale, we find by means of (3.38) and (3.19) that

$$
d Z_{t}^{s}=\kappa \frac{\partial P}{\partial h}\left(T-t, \tilde{H}_{t}\right)(-\delta) \tilde{H}_{t} d \tilde{W}_{t}=\sigma u_{t}^{s} Z_{t}^{s} d \tilde{W}_{t}
$$

for $t \in\left[t_{0}, T\right]$. It follows from the second identity in (3.42) that

$$
\begin{aligned}
u_{t}^{s} & =-\frac{\delta}{\sigma} \kappa \frac{\partial P}{\partial h}\left(T-t, \tilde{H}_{t}\right) \frac{\tilde{H}_{t}}{Z_{t}^{s}}=-\frac{\delta}{\sigma} \frac{x_{0}-e^{-r\left(T-t_{0}\right)} g}{\mathrm{E}\left[\tilde{H}_{T}\left(\lambda-\tilde{H}_{T}\right)^{+}\right]} \frac{\partial P}{\partial h}\left(T-t, \tilde{H}_{t}\right) \frac{\tilde{H}_{t}}{Z_{t}^{s}} \\
& =-\frac{\delta}{\sigma} \frac{e^{r\left(t-t_{0}\right)} x_{0}-e^{-r(T-t)} g}{P\left(T-t_{0}, 1\right)} \frac{\partial P}{\partial h}\left(T-t, \tilde{H}_{t}\right) \frac{\tilde{H}_{t}}{X_{t}^{s}}
\end{aligned}
$$

for $t \in\left[t_{0}, T\right]$. Note that the second identity in (3.4) can be used to express $(\partial P / \partial h)(T-t, h)$ in closed form using the standard normal distribution function $\Phi$, however, we will omit the 
explicit expression for simplicity. Note also that $h \mapsto P(T-t, h)$ is strictly increasing (and continuous) so that from (3.39) we see that

$$
\begin{aligned}
\tilde{H}_{t} & =P_{h}^{-1}\left(T-t,\left(e^{r(T-t)} X_{t}^{s}-g\right) / \kappa\right) \\
& =P_{h}^{-1}\left(T-t, \frac{e^{r(T-t)} X_{t}^{s}-g}{x_{0}-e^{-r\left(T-t_{0}\right)} g} \mathrm{E}\left[\tilde{H}_{T}\left(\lambda-\tilde{H}_{T}\right)^{+}\right]\right) \\
& =P_{h}^{-1}\left(T-t, \frac{e^{r(T-t)} X_{t}^{s}-g}{e^{r\left(T-t_{0}\right)} x_{0}-g} P\left(T-t_{0}, 1\right)\right)
\end{aligned}
$$

for $t \in\left[t_{0}, T\right]$. Inserting $\tilde{H}_{t}$ into the right-hand side of (3.43), we find that the statically optimal control in (2.6) is given by

$$
\begin{aligned}
u_{*}^{s}(t, x)= & -\frac{\delta}{\sigma} \frac{1}{x} \frac{e^{r\left(t-t_{0}\right)} x_{0}-e^{-r(T-t)} g}{P\left(T-t_{0}, 1\right)} \\
& \times \frac{\partial P}{\partial h}\left(T-t, P_{h}^{-1}\left(T-t, \frac{e^{r(T-t)} x-g}{e^{r\left(T-t_{0}\right)} x_{0}-g} P\left(T-t_{0}, 1\right)\right)\right) \\
& \times P_{h}^{-1}\left(T-t, \frac{e^{r(T-t)} x-g}{e^{r\left(T-t_{0}\right)} x_{0}-g} P\left(T-t_{0}, 1\right)\right)
\end{aligned}
$$

for $(t, x) \in\left[t_{0}, T\right] \times \mathbb{R}$ such that $e^{-r(T-t)} g<x<e^{-r(T-t)} \beta$. (If $x=e^{-r(T-t)} g$ or $x \geq e^{-r(T-t)} \beta$ then $u_{*}^{d}(t, x)=0$ as discussed prior to Definition 1 above.)

7. The statically optimal wealth process $X^{s}$ can be characterised as the unique strong solution to the stochastic differential equation (2.3) obtained by inserting the statically optimal control $u_{t}^{s}=u_{*}^{s}\left(t, X_{t}^{s}\right)$ in place of $u_{t}$ for $t \in\left[t_{0}, T\right]$. From (3.27) we see that

$$
X_{T}^{s}=\kappa(\lambda-H)^{+}+g
$$

where $\lambda=\lambda\left(T-t_{0}, x_{0}\right)$ is the unique strictly positive solution to (3.31) and $\kappa=\kappa\left(T-t_{0}, x_{0}\right)$ is given by (3.30) with $\mathrm{P}=\mathrm{P}_{t_{0}, x_{0}}, H=H_{T} / H_{t_{0}}$ and $x=x_{0}$. The static value function is given by

$$
V_{s}\left(t_{0}, x_{0}\right)=\operatorname{Var}_{t_{0}, x_{0}}\left(X_{T}^{s}\right)=\left(\kappa\left(t_{0}, x_{0}\right)\right)^{2} \operatorname{Var}\left[\left(\lambda\left(t_{0}, x_{0}\right)-H_{T-t_{0}}\right)^{+}\right] .
$$

Note that the latter variance can be readily calculated, however, we will omit the explicit expression for simplicity.

8. To obtain a candidate for the dynamically optimal control $u_{*}^{d}$ at $(t, x) \in\left[t_{0}, T\right] \times \mathbb{R}$ given and fixed, we will identify $t_{0}$ with $t$, and $x_{0}$ with $x$, in the statically optimal control $u_{*}^{s}(t, x)$ given in (3.45) above. This yields $\tilde{H}_{t}=H_{t} / H_{t_{0}}=1$ and a direct differentiation of the right-hand side in (3.40) shows that

$$
\frac{\partial P}{\partial h}(T-t, 1)=-e^{r(T-t)} \mathrm{E}\left[H_{T-t}^{2} I\left(\lambda>H_{T-t}\right)\right]
$$

for $t \in\left[t_{0}, T\right]$. Inserting (3.48) into (3.45), with $\left(t_{0}, x_{0}\right)=(t, x)$ and $\tilde{H}_{t}=1$, upon recalling $(3.3)+(3.4)$ and noting from (3.40) that

$$
P(T-t, 1)=e^{r(T-t)} \mathrm{E}\left[H_{T-t}\left(\lambda-H_{T-t}\right)^{+}\right]
$$


we see that a candidate for the dynamically optimal control in (2.6) is given by

$$
u_{*}^{d}(t, x)=\frac{\delta}{\sigma} \frac{1}{x}\left(x-e^{-r(T-t)} g\right) a(T-t, x)
$$

(as stated in (3.6) above) where $a(T-t, x)$ is given in (3.7), with $\lambda=\lambda(T-t, x)$ being a unique strictly positive solution to the equation (3.8), for $(t, x) \in\left[t_{0}, T\right] \times \mathbb{R}$ given and fixed (as claimed in Part (A) of the theorem).

To see that the control $u_{*}^{d}$ is dynamically optimal in the sense of Definition 2 , take any control $v$ from $C_{g, \beta}$ such that $v(t, x) \neq u_{*}^{d}(t, x)$ for $(t, x) \in\left[t_{0}, T\right] \times \mathbb{R}$ given and fixed, and choose $w$ in $C_{g, \beta}$ to be statically optimal control under the measure $\mathrm{P}_{t, x}$. Then $w(t, x)=$ $u_{*}^{s}(t, x)=u_{*}^{d}(t, x) \neq v(t, x)$ so that by continuity of $w$ and $v$ we can conclude that $w(s, y) \neq$ $v(s, y)$ for all $(s, y) \in[t, t+\varepsilon] \times[x-\varepsilon, x+\varepsilon]$ for some $\varepsilon>0$ sufficiently small. But then by the uniqueness part of the Itô-Clark theorem, referred to following (3.23) above, we can conclude that $\mathrm{P}_{t, x}\left(X_{T}^{v} \neq X_{T}^{w}\right)>0$, where we recall that $X_{T}^{w}=X_{*}$ under $\mathrm{P}_{t, x}$ with $X_{*}$ given in (3.27). Since moreover $X_{*}$ has been seen to be a unique point of the minimum in (3.26) up to a set of $\mathrm{P}_{t, x}$-measure zero, it thus follows from (3.14) and (3.28) that

$$
\operatorname{Var}_{t, x}\left(X_{T}^{w}\right)=\mathrm{E}_{t, x}\left[\left(X_{T}^{w}\right)^{2}\right]-\beta^{2}<\mathrm{E}_{t, x}\left[\left(X_{T}^{v}\right)^{2}\right]-\beta^{2}=\operatorname{Var}_{t, x}\left(X_{T}^{v}\right)
$$

as needed in Definition 2. This shows that the control $u_{*}^{d}$ from (3.50) is dynamically optimal in (2.6) as claimed in Part (A) of the theorem.

(B): Inserting the dynamically optimal control $u_{*}^{d}$ into (2.3) we find that the dynamically optimal wealth process $X^{d}$ solves the stochastic differential equation (3.9) under the initial condition $X_{t_{0}}^{d}=x_{0} \in\left(e^{-r\left(T-t_{0}\right)} g, e^{-r\left(T-t_{0}\right)} \beta\right)$. By Itô's existence and uniqueness result for locally Lipschitz coefficients (see e.g. [19, pp 132-132]) we know that the stochastic differential equation (3.9) has a unique strong solution until the first exit time of $X_{t}^{d}$ from the interval $\left(e^{-r(T-t)} g, e^{-r(T-t)} \beta\right)$ when $t$ runs from $t_{0}$ to $T$ followed by the constant motion corresponding to $u_{*}^{d}=0$ afterwards (as discussed prior to Definition 1 above).

9. In order to analyse the hitting times $\tau_{\beta}$ and $\tau_{g}$ of $X^{d}$ to the upper and lower boundaries as defined in (3.10) and (3.11) respectively, we will consider the process $Z$ defined by

$$
Z_{t}:=e^{r(T-t)} X_{t}^{d}
$$

for $t \in\left[t_{0}, T\right]$ (omitting superscript $d$ to $Z$ to simplify the notation). From (3.6)-(3.8) and (3.19) we see that $Z$ solves

$$
d Z_{t}=\delta\left(Z_{t}-g\right) A\left(T-t, Z_{t}\right) d \tilde{W}_{t}
$$

under $\tilde{\mathrm{P}}_{t_{0}, z_{0}}$ for $z_{0} \in(g, \beta)$ where the function $A$ is defined by

$$
A(T-t, z):=a\left(T-t, e^{-r(T-t)} z\right)=\frac{\mathrm{E}\left[H_{T-t}^{2} I\left(\lambda>H_{T-t}\right)\right]}{\mathrm{E}\left[H_{T-t}\left(\lambda-H_{T-t}\right)^{+}\right]}
$$

and $\lambda=\lambda\left(T-t, e^{-r(T-t)} z\right)$ is the unique strictly positive solution to (3.8) which reads

$$
\frac{\mathrm{E}\left[H_{T-t}\left(\lambda-H_{T-t}\right)^{+}\right]}{\mathrm{E}\left[\left(\lambda-H_{T-t}\right)^{+}\right]}=e^{-r(T-t)} \frac{z-g}{\beta-g}
$$


for $(t, z) \in\left[t_{0}, T\right] \times[g, \beta]$.

10. Note that $Z$ is a bounded martingale and hence $\tilde{\mathrm{P}}_{t_{0}, z_{0}}$-a.s. convergent as $t \uparrow T$ for $z_{0} \in(g, \beta)$. If its limit $Z_{T}$ would belong to $(g, \beta)$, then using $(3.33)$ we would have $\lambda(T-$ $\left.t, e^{-r(T-t)} Z_{t}\right) \rightarrow \lambda\left(0+, Z_{T}\right) \in(0, \infty) \quad \tilde{\mathrm{P}}_{t_{0}, z_{0}}$-a.s. as $t \uparrow T$, so that the left-hand side of (3.55) would tend to 1 , while its right-hand side would tend to $\left(Z_{T}-g\right) /(\beta-g) \in(0,1)$, which is a contradiction. We can therefore conclude that $Z_{t} \rightarrow Z_{T} \in\{g, \beta\} \quad \tilde{\mathrm{P}}_{t_{0}, z_{0}}$-a.s. as $t \uparrow T$.

11. We now show that $Z$ does not hit $\beta$ strictly before $T$ with a strictly positive probability as claimed in the first equality of (3.12). For this, we first establish that the following limiting relation is satisfied

$$
\begin{aligned}
\lim _{z \uparrow \beta} \frac{A(T-t, z)}{\beta-z} & =\frac{1}{\beta-g} \lim _{\lambda \uparrow \infty}\left(\frac{\frac{\mathrm{E}\left[H_{T-t}^{2} I\left(\lambda>H_{T-t}\right)\right]}{\mathrm{E}\left[H_{T-t}\left(\lambda-H_{T-t}\right)^{+}\right]}}{1-e^{r(T-t)} \frac{\mathrm{E}\left[H_{T-t}\left(\lambda-H_{T-t}\right)^{+}\right]}{\mathrm{E}\left[\left(\lambda-H_{T-t}\right)^{+}\right]}}\right) \\
& =\frac{\mathrm{E}\left(H_{T-t}^{2}\right)}{(\beta-g) \mathrm{E}\left(H_{T-t}\right)\left[e^{r(T-t)} \mathrm{E}\left(H_{T-t}^{2}\right)-\mathrm{E}\left(H_{T-t}\right)\right]}
\end{aligned}
$$

where in the first equality we use (3.54) and the fact that $\beta-z=(\beta-g)\left[1-e^{r(T-t)} F(\lambda)\right]$, upon letting $F(\lambda)$ denote the left-hand side in (3.55), combined with $\lambda=\lambda\left(T-t, e^{-r(T-t)} z\right) \uparrow \infty$ as $z \uparrow \beta$ which is seen from (3.55) using (3.33) above, and the second equality follows by calculating the limit upon making use of the fact that the probability density function of $H_{T-t}$ tends faster to zero than any polynomial (of third degree) when the argument tends to infinity. (Details of these lengthy but elementary calculations are omitted for simplicity.) Moreover, the convergence in (3.56) is uniform over $t \in\left[t_{0}, T_{1}\right]$ so that finiteness of the limit in (3.56) implies that the function $\tilde{A}$ defined by

$$
\tilde{A}(T-t, z):=(z-g) \frac{A(T-t, z)}{\beta-z}
$$

is bounded for $(t, z) \in\left[t_{0}, T_{1}\right] \times(g, \beta)$ as is easily seen that $(z-g) A(T-t, z) \rightarrow 0$ as $z \downarrow g$ and this convergence is uniform over $t \in\left[t_{0}, T_{1}\right]$ for any $T_{1} \in\left[t_{0}, T\right)$ given and fixed. From (3.53) we see that $Z$ solves

$$
d Z_{t}=\delta\left(\beta-Z_{t}\right) \tilde{A}\left(T-t, Z_{t}\right) d \tilde{W}_{t}
$$

so that by setting $Y_{t}:=\beta-Z_{t}$ we see that $Y$ solves

$$
d Y_{t}=\delta Y_{t} \tilde{A}\left(T-t, \beta-Y_{t}\right) d \hat{W}_{t}
$$

where we set $\hat{W}=-\tilde{W}$. From (3.59) we find that

$$
Y_{t}=Y_{t_{0}} \exp \left[\int_{t_{0}}^{t} \tilde{A}\left(T-s, \beta-Y_{s}\right) d \hat{W}_{s}-\frac{1}{2} \int_{t_{0}}^{t} \tilde{A}^{2}\left(T-s, \beta-Y_{s}\right) d s\right]
$$

with $\int_{t_{0}}^{t} \tilde{A}^{2}\left(T-s, \beta-Y_{s}\right) d s<\infty$ for $t \in\left[t_{0}, T_{1}\right]$ by the boundedness of $\tilde{A}$. Hence from (3.60) we see that $Y_{t}>0$ i.e. $Z_{t}<\beta \quad \tilde{\mathrm{P}}_{t_{0}, z_{0}}$-a.s. for all $t \in\left[t_{0}, T_{1}\right]$ with $T_{1} \in\left[t_{0}, T\right)$ and $z_{0} \in(g, \beta)$, showing that $Z$ does not hit $\beta$ strictly before $T$ with a strictly positive probability, as claimed 
in the first equality in (3.12). The second equality in (3.12) follows from the fact that $Z$ is a bounded martingale converging $\tilde{\mathrm{P}}_{t_{0}, z_{0}}$-a.s. to either $g$ or $\beta$ as $t \uparrow T$ for $z_{0} \in(g, \beta)$, so that $Z$ must hit $\beta$ at time $T$ with a strictly positive probability as claimed.

12. We finally show that $Z$ hits $g$ strictly before $T$ with a strictly positive probability as claimed in (3.13). For this, we first establish that the following limiting relation is satisfied

$$
\lim _{z \downarrow g} \frac{A(T-t, z)}{|\log (z-g)|}=\lim _{\lambda \downarrow 0} \frac{\mathrm{E}\left[H_{T-t}^{2} I\left(\lambda>H_{T-t}\right)\right]}{|\log (\lambda)| \mathrm{E}\left[H_{T-t}\left(\lambda-H_{T-t}\right)^{+}\right]}=1
$$

where in the first equality we use that $\log (z-g)=\log (\beta-g)+r(T-t)+\log (F(\lambda))$, upon letting $F(\lambda)$ denote the left-hand side in (3.55), combined with $\lambda=\lambda\left(T-t, e^{-r(T-t)} z\right) \downarrow 0$ as $z \downarrow g$ which is seen from (3.55) using (3.33) above, and that

$$
\lim _{\lambda \downarrow 0} \frac{F(\lambda)}{\lambda}=\lim _{\lambda \downarrow 0} \frac{\mathrm{E}\left[H_{T-t}\left(\lambda-H_{T-t}\right)^{+}\right]}{\lambda \mathrm{E}\left[\left(\lambda-H_{T-t}\right)^{+}\right]}=1
$$

upon making use of the fact that $\log \left(H_{T-t}\right)$ is normally distributed to establish the final equalities in (3.61) and (3.62). (Details of these lengthy but elementary calculations are omitted for simplicity.) Moreover, the convergence in (3.61) is uniform over $t \in\left[t_{0}, T_{1}\right]$ so that the strict positivity and finiteness of the limit in (3.61) imply that the function $\tilde{A}$ defined by

$$
\tilde{A}(T-t, z):=\frac{A(T-t, z)}{|\log (z-g)|}
$$

is bounded away from zero and bounded from above for $(t, z) \in\left[t_{0}, T_{1}\right] \times(g, \beta)$ with any $T_{1} \in\left[t_{0}, T\right)$ given and fixed. From (3.53) we see that $Z$ solves

$$
d Z_{t}=\delta\left(Z_{t}-g\right)\left|\log \left(Z_{t}-g\right)\right| \tilde{A}\left(T-t, Z_{t}\right) d \tilde{W}_{t}
$$

so that by setting $Y_{t}:=Z_{t}-g$, we see that $Y$ solves

$$
d Y_{t}=\delta Y_{t}\left|\log Y_{t}\right| \tilde{A}\left(T-t, g+Y_{t}\right) d \tilde{W}_{t} .
$$

Defining the additive functional

$$
C_{t}:=\int_{t_{0}}^{t} \tilde{A}^{2}\left(T-s, g+Y_{s}\right) d s
$$

and letting $S_{t}:=C_{t}^{-1}$, we find that the time-changed process $\hat{Y}_{t}:=Y_{S_{t}}$ solves

$$
d \hat{Y}_{t}=\delta \hat{Y}_{t}\left|\log \hat{Y}_{t}\right| d \hat{W}_{t}
$$

where $\hat{W}_{t}=\int_{t_{0}}^{t} \tilde{A}\left(T-S_{s}, g+Y_{S_{s}}\right) d \tilde{W}_{S_{s}}$ defines a standard Brownian motion for $t \in\left[t_{0}, T\right]$, as is easily seen by Lévy's characterisation theorem. The 'logarithmic' diffusion process $\hat{Y}$ solving (3.67) is on natural scale (local martingale), so that the scale function of $\hat{Y}$ is given by $S(y)=y$ for $y \in(0, \infty)$. Since $S(\infty)=\infty$ we see that the explosion of $\hat{Y}$ (i.e. its exit from $(0, \infty))$ in finite time is only possible at 0 . Moreover, we see that

$$
v(y):=\int_{1}^{y} S^{\prime}(z)\left(\int_{1}^{z} \frac{2 d w}{S^{\prime}(w) \sigma^{2}(w)}\right) d z=\int_{1}^{y} \int_{1}^{z} \frac{2 d w d z}{\delta^{2} w^{2} \log ^{2} w}
$$


for $y \in(0, \infty)$ where $\sigma(w):=\sigma w|\log w|$ for $w \in(0, \infty)$. A direct examination of the final (double) integral in (3.68) shows that

$$
v(0+)<\infty \quad \& \quad v(\infty)=\infty
$$

Therefore by Feller's test (see [9, Proposition 5.32, p. 350]) we can conclude that the explosion of $\hat{Y}$ at zero occurs with probability one (i.e. $\hat{Y}$ hits 0 with probability one). Since $\hat{Y}$ is a regular time-homogenous diffusion process, it follows therefore that $\hat{Y}$ hits zero with a strictly positive $\tilde{\mathrm{P}}_{t_{0}, y_{0}}$-probability before any given deterministic time $t_{1} \in\left(0, T-t_{0}\right]$ has elapsed. Using the fact that $\tilde{A}(T-t, z)$ is bounded away from zero for $(t, z) \in\left[t_{0}, T_{1}\right] \times(g, \beta)$ with any $T_{1} \in\left[t_{0}, T\right)$ given and fixed, from (3.66) we see that $C_{t} \geq c\left(t-t_{0}\right)=: t_{1}$ for $t \in\left(t_{0}, T_{1}\right]$ with some $c>0$. This shows that $C_{t}$ is greater than or equal to the first hitting time of $\hat{Y}$ to 0 with a strictly positive probability. Since 0 is an exit (not entrance) boundary point for $\hat{Y}$, this shows that $\hat{Y}_{t_{0}+C_{t}}=Y_{t}=0$ with a strictly positive probability for $t \in\left(t_{0}, T_{1}\right]$. Thus $Z$ hits $g$ strictly before $T$ with a strictly positive probability as claimed in (3.13) and the proof of the theorem is complete.

Remark 4. The proof above shows that there exists a unique pair $\left(\lambda_{1}, \lambda_{2}\right)$ in $\mathbb{R}^{2}$ such that $X_{*}$ from (3.27) solves $(3.24)+(3.25)$ where $\lambda_{2}$ is always strictly negative (while $\lambda_{1}$ is real valued). For this reason we have imposed following (3.27) that $\kappa>0$ i.e. $\lambda_{2}<0$ and sought the solution $\left(\lambda_{1}, \lambda_{2}\right)$ to $(3.28)+(3.29)$ in $\mathbb{R} \times(-\infty, 0)$. The arguments presented in parts 3 and 4 of the proof above establish the existence of a unique pair $\left(\lambda_{1}, \lambda_{2}\right)$ in $\mathbb{R} \times(-\infty, 0)$ such that $X_{*}$ solves $(3.24)+(3.25)$. To complete the proof of the initial claim above, it is thus enough to show that there is no $\lambda_{2} \geq 0$ and $\lambda_{1} \in \mathbb{R}$ such that (3.28) and (3.29) hold with $+H$ in place of $-H$, where $\kappa=\lambda_{2} / 2$ and $\lambda=\left(\lambda_{1} / 2-g\right) /\left(\lambda_{2} / 2\right)$. This verification can be made along the same lines as in the proof above. Indeed, defining $F$ as in (3.32) with $+H$ in place $-H$, it is easily seen using the same arguments as above that $\lambda \mapsto F(\lambda)$ is (strictly) decreasing on $(-\infty, 0]$. Denoting the right-hand side in (3.31) by $R$, and noting that $R \in(0, \mathrm{E}(H))$, we thus see that if equality in (3.31) would hold for some $\lambda \leq 0$, then we would have $\mathrm{E}\left(H^{2}\right) / \mathrm{E}(H)=F(0) \leq F(\lambda)=R<\mathrm{E}(H)$, which would violate Jensen's inequality. Similarly, if equality in (3.31) would hold for some $\lambda>0$, then solving for $\lambda$ in (3.31) we would get $\lambda=-\left(\mathrm{E}\left(H^{2}\right)-\mathrm{E}(H) R\right) /(\mathrm{E}(H)-R)>0$, which would again violate Jensen's inequality due to $R<\mathrm{E}(H)$. Combined with the arguments recalled above this shows that there exists a unique pair $\left(\lambda_{1}, \lambda_{2}\right)$ in $\mathbb{R}^{2}$ such that $X_{*}$ solves $(3.24)+(3.25)$ as claimed.

Moreover, it may be noted that $X_{*}$ is almost surely a pointwise unique solution to (3.24)+ (3.25). Indeed, if $Z_{*}$ solves (3.24) $+(3.25)$ then denoting the value in (3.24) by $V$, we see that $V=\mathrm{E}\left(Z_{*}^{2}\right)=\mathrm{E}\left(Z_{*}^{2}-\lambda_{1} Z_{*}-\lambda_{2} H Z_{*}\right)+\lambda_{1} \beta+\lambda_{2} x \geq \mathrm{E}\left(X_{*}^{2}-\lambda_{1} X_{*}-\lambda_{2} H X_{*}\right)+\lambda_{1} \beta+\lambda_{2} x=\mathrm{E}\left(X_{*}^{2}\right)=V$, from where we infer that if the inequality $Z_{*}^{2}-\lambda_{1} Z_{*}-\lambda_{2} H Z_{*} \geq X_{*}^{2}-\lambda_{1} X_{*}-\lambda_{2} H X_{*}$ would be strict with a strictly positive probability, then we would have $V>V$ which is a contradiction. Hence $Z_{*}=X_{*}$ almost surely by the uniqueness of the minimum point $X_{*}$ of $x \mapsto x^{2}-\lambda_{1} x-\lambda_{2} H x$ for $x \geq g$ as claimed.

Remark 5. Note that one can extract the statically optimal control $u_{*}^{s}$, the statically optimal controlled wealth process $X^{s}$, and the static value function $V_{s}$ from the proof above. They are given by (3.45), (3.41) and (3.47) respectively (compare these expressions with the pathwise unconstrained ones given in $[16$, Corollary 7 , Part A]). Note, in particular, that in 
addition to depending explicitly on the initial time $t_{0}$ and the initial wealth $x_{0}$, the statically optimal control $u_{*}^{s}$ is expressed explicitly in terms of the statically optimal controlled wealth process $X_{t}^{s}$ evaluated at the current time $t$ (and the current time $t$ itself).

The dynamically optimal control $u_{*}^{d}$ from (3.6) by its nature rejects any past point $\left(t_{0}, x_{0}\right)$ to measure its performance, so that any comparison between the static value $V_{s}\left(t_{0}, x_{0}\right)$ and the dynamic value $V_{d}\left(t_{0}, x_{0}\right)$ is meaningless from the standpoint of the dynamic optimality. Another issue with a plain comparison between the values $V_{s}(t, x)$ and $V_{d}(t, x)$ for $(t, x) \in$ $\left[t_{0}, T\right] \times \mathbb{R}$ is that the optimally controlled processes $X^{s}$ and $X^{d}$ may never come to the same point $x$ at the same time $t$, so that the comparison itself may be unreal. A more dynamic way, which also makes more sense in general, is to compare the value functions composed with the controlled processes. This amounts to look at $V_{s}\left(t, X_{t}^{s}\right)$ and $V_{d}\left(t, X_{t}^{d}\right)$ for $t \in\left[t_{0}, T\right]$ and pay particular attention to $t$ becoming the terminal value $T$.

Recalling that $X_{T}^{d}$ takes only values $g$ and $\beta$, and noting that $V_{d}(t, g)=V_{d}(t, \beta)=0$ for all $t \in\left[t_{0}, T\right]$, we see that $V_{d}\left(T, X_{T}^{d}\right)=0$. On the other hand, it is easily seen from (3.31) with $H=H_{T} / H_{t}$ that $\lambda=\lambda\left(T-t, X_{t}^{s}\right) \rightarrow 0$ as $t \uparrow T$ when $X_{T}^{s} \in(g, \beta)$. From $(3.27)+(3.28)$ and $(3.46)+(3.47)$, we therefore find using the first equality in (3.30) and L'Hospital's rule that

$$
\begin{aligned}
V_{s}\left(t, X_{t}^{s}\right) & =\operatorname{Var}_{t, X_{t}^{s}}\left(X_{T}^{s}\right)=\mathrm{E}\left[\left(\kappa(\lambda-H)^{+}+g\right)^{2}\right]-\beta^{2} \\
& =(\beta-g)^{2}\left[\frac{\mathrm{E}\left[\left((\lambda-H)^{+}\right)^{2}\right]}{\left(\mathrm{E}\left[(\lambda-H)^{+}\right]\right)^{2}}-1\right] \longrightarrow \infty
\end{aligned}
$$

as $t \uparrow T$, whenever $X_{T}^{s} \in(g, \beta)$ due to $\lambda=\lambda\left(T-t, X_{t}^{s}\right) \rightarrow 0$ and $H=H_{T} / H_{t} \rightarrow 1$. Form (3.46) we however see that $X_{T}^{s} \in(g, \beta)$ with a strictly positive $\mathrm{P}_{t_{0}, x_{0}}$-probability, so that $\mathrm{E}_{t_{0}, x_{0}}\left[V_{s}\left(t, X_{t}^{s}\right)\right] \rightarrow \infty$ as $t \uparrow T$. It follows therefore that

$$
E_{t_{0}, x_{0}}\left[V_{s}\left(T, X_{T}^{s}\right)\right]=\infty>0=E_{t_{0}, x_{0}}\left[V_{d}\left(T, X_{T}^{d}\right)\right]
$$

for $\left(t_{0}, x_{0}\right) \in[0, T] \times \mathbb{R}$ such that $e^{-r\left(T-t_{0}\right)} g<x_{0}<e^{-r\left(T-t_{0}\right)} \beta$. This shows that the dynamic control $u_{*}^{d}$ from (3.6) outperforms the static control $u_{*}^{s}$ from (3.45) in the pathwise constrained problem (2.6).

\section{References}

[1] Basak, S. and Chabakauri, G. (2010). Dynamic mean-variance asset allocation. Rev. Financ. Stud. 23 (2970-3016).

[2] Basak, S. and Chabakauri, G. (2012). Dynamic hedging in incomplete markets: A simple solution. Rev. Financ. Stud. 25 (1845-1896).

[3] Chabakauri, G. and Han, B. Y. (2017). Collateral requirements and asset prices. Working Paper, London Scool of Economics.

[4] Bi, J. Meng, Q. and Zhang, Y. (2014). Dynamic mean-variance and optimal reinsurance problems under the no-bankruptcy constraint for an insurer. Ann. Oper. Res. 212 (43-59).

[5] Bielecki, T. R. Jin, H. Pliska, S. R. and Zhou X. Y. (2005). Continuous-time mean-variance portfolio selection with bankruptcy prohibition. Math. Finance 15 (213244). 
[6] BJöRK, T. (2009). Arbitrage Theory in Continuous Time. Oxford Univ. Press.

[7] He, H. and PAGÈs, H. F. (1993). Labor income, borrowing constraints, and equilibrium asset prices. Econom. Theory 3 (663-696).

[8] Hoffmann-Jørgensen, J. (1994). Probability with a View Toward Statistics (Vol I). Chapman \& Hall.

[9] Karatzas, I. and Shreve, S. E. (1991). Brownian Motion and Stochastic Calculus. Springer-Verlag.

[10] Korn, R. (2005). Optimal portfolios with a positive lower bound on final wealth. Quant. Finance 5 (315-321).

[11] Korn, R. and Trautmann, S. (1995). Continuous-time portfolio optimization under terminal wealth constraints. ZOR Math. Methods Oper. Res. 42 (69-92).

[12] LI, D. and NG, W. L. (2000). Optimal dynamic portfolio selection: Multiperiod meanvariance formulation. Math. Finance 10 (387-406).

[13] Li, X. Zhou, X. Y. and Lim, A. E. B. (2002). Dynamic mean-variance portfolio selection with no-shorting constraints. SIAM J. Control Optim. 40 (1540-1555).

[14] Markowitz, H. M. (1952). Portfolio selection. J. Finance 7 (77-91).

[15] Merton, R. C. (1972). An analytic derivation of the efficient portfolio frontier. J. Financial Quant. Anal. 7 (1851-1872).

[16] Pedersen, J. L. and Peskir, G. (2017). Optimal mean-variance portfolio selection. Math. Financ. Econ. 11 (137-160).

[17] PliskA, S. R. (1986). A stochastic calculus model of continuous trading: Optimal portfolios. Math. Oper. Res. 11 (370-382).

[18] Richardson, H. R. (1989). A minimum variance result in continuous trading portfolio optimization. Management Sci. 35 (1045-1055).

[19] Rogers, L. C. G. and Williams, D. (2000). Diffusions, Markov Processes and Martingales: Itô Calculus (Vol 2). Cambridge University Press.

[20] Strotz, R. H. (1956). Myopia and inconsistency in dynamic utility maximization. Rev. Econ. Stud. 23 (165-180).

[21] White, D. J. (1974). Dynamic programming and probabilistic constraints. Operations Res. 22 (654-664).

[22] Zhou, X. Y. and Li, D. (2000). Continuous-time mean-variance portfolio selection: A stochastic LQ framework. Appl. Math. Optim. 42 (19-33).

Jesper Lund Pedersen

Department of Mathematical Sciences

University of Copenhagen

Universitetsparken 5

DK-2100 Copenhagen

Denmark

jesper@math.ku.dk
Goran Peskir

School of Mathematics

The University of Manchester

Oxford Road

Manchester M13 9PL

United Kingdom

goran@maths.man.ac.uk 